\title{
GRAIN REFINEMENT OF TiAl ALLOYS \\ BY ISOMORPHIC SELF-INOCULATION
}

\author{
J.R. Kennedy ${ }^{1,2,3}$, D. Daloz ${ }^{1,2}$, B. Rouat ${ }^{1,2}$, E. Bouzy ${ }^{2,3}$, J. Zollinger ${ }^{1,2}$ \\ 1 Université de Lorraine, Institut Jean Lamour, \\ Department of Metallurgy and Materials Science and Engineering, Nancy, France \\ 2 Laboratory of Excellence on Design of Alloy Metals for low-mAss Structures \\ ('LabEx DAMAS'), France
}

3 Université de Lorraine, LEM3, Metz, France

\begin{abstract}
Grain refinement by inoculation relies upon particles which act as heterogeneous nucleation sites. A novel concept of grain refinement by isomorphic self-inoculation is introduced in this paper. The inoculant particles have the same crystallographic structure as the solidifying phase, and the nucleation stage is replaced by direct epitaxial growth compared to classical inoculation. This concept has been successfully applied to a Ti-Al alloy, where classical inoculants (borides, carbides) can be embrittling in the as-cast state. Casting trials were successful in reducing the ascast grain size as well as increasing the equiaxed grain fraction. It is shown that, opposed to classical inoculation theory, the particle size distribution has no influence and only the number of inoculant particles introduced impacts the final grain size. Moreover, the results suggest that each introduced particle can be responsible for multiple grains found in the as-cast ingot.
\end{abstract}

\section{KEYWORDS}

Titanium Aluminides, Grain Refinement, Solidification, Inoculation 


\section{Introduction}

The microstructure of a material has a drastic effect on its properties and industrial applications. Reducing the grain size of a given alloy can improve its properties and allows it to be used in applications not previously viable. Inoculation is a method of grain refinement by increasing the number of grains formed during solidification and is used extensively in industry, especially for aluminum alloys [1]. This is achieved by increasing the number of nucleation sites present in the melt. Powders can be added which are effective nuclei or alloying additions can be made which will form precipitates in the melt and act as nucleation sites [2]. In order for an inoculant to be effective it must have a low lattice misfit between itself and the nucleated phase [3], be stable enough in the melt to survive until solidification, not have negative effects on the final properties of the material, and have good usability. An inoculant with good usability does not agglomerate or have excessive settling in the melt, can be easily introduced into the melt, and is effective at low addition levels. Inoculation is of particular interest for titanium aluminum alloys which have recently begun being used in the last low pressure stage of jet turbines. With grain refinement these alloys could potentially be used further, into hotter sections of turbines.

The high temperature oxidation resistance and high specific strength make Ti-Al Alloys particularly appealing for aerospace applications [4]. The Ti-Al alloys of interest for these applications normally fall on the peritectic plateau at 44-48 at $\% \mathrm{Al}$ [5]. The solidification path for alloys in this composition range is complex and can include up to four phase changes from liquid to room temperature [5]. These could include the initial solidification of $\beta$ grains from the liquid, transformation of $\beta$ grains to $\alpha$ (following Burgers orientation relationship (110) $\left.\beta / /(0001)_{\alpha}[6]\right), \alpha$ to $\alpha$ and $\gamma$ (following Blackburn's orientation relationship (0001) $\left.\alpha / /(111)_{\gamma}[7]\right)$ and finally $\alpha$ and $\gamma$ to $\gamma$ and $\alpha_{2}$ [8]. Grain refinement can be attempted during any of these phase transformations. Attempts to grain refine by using the $\gamma$ massive transformation during heat treatment requires large cooling rates which result in deformations that make applications of the method difficult for manufacturing parts $[9,10]$. The most ideal location of grain refinement during processing is then the initial solidification of $\beta$ grains from the liquid. This is most commonly achieved by inoculating Ti-Al alloys via boron additions. Relatively small amounts of boron are added to the melt and titanium boride $(\mathrm{TiB})$ or diboride $\left(\mathrm{TiB}_{2}\right)$ precipitate as nucleants. These precipitates can nucleate 
$\alpha$ or $\beta$ phases depending on alloy composition, and have been found to have orientation relationships with both phases, which indicates a good lattice mismatch between them [11], they are also thermodynamically stable in the melt [12]. However, in some cases the borides which form can have flake [13] or needle like [14] morphologies. These morphologies are detrimental to the final mechanical properties of the alloy and can result in decreased creep resistance and tensile strength, leading to brittle fracture [15]. These precipitates function as traditional inoculants following the Laplace-Young equation [2]:

$\Delta T_{g}=\frac{4 \Gamma_{s l} \sin \theta}{\phi}$

where $\theta$ is the contact angle between the nucleus and inoculant particle, $\varphi$ is effectively the inoculant particle size and $\Delta \mathrm{T}_{\mathrm{g}}$ is the undercooling required. $\Gamma_{\mathrm{sl}}$ is the Gibbs-Thompson coefficient, $\Gamma_{s l}=2 V_{m}^{s} \sigma_{s l} / \Delta S_{m}^{f}$, where $V_{m}^{s}$ is the molar volume of the solid phase, $\sigma_{s l}$ is the surface tension and $\Delta S_{m}^{f}$ is the molar entropy of fusion. This shows that as the particle size decreases a greater undercooling is required for nucleation and thus the ability of the particles to successfully inoculate the melt is highly dependent on their size [16].

A novel concept is proposed to inoculate Ti-Al alloys using an inoculant made from the solidifying Ti-Al phase a process here dubbed isomorphic self-inoculation. A similar method of grain refining low alloy $\mathrm{Ti}$ with pure $\mathrm{Ti}$ powders has been proposed by Bermingham et al. [17]. In their experiments particles of pure Ti were placed above a mold and molten Ti was poured over them, into the mold to solidify. These particles were carried into the solidifying melt and acted as heterogeneous nucleation sites. For isomorphic self-inoculation an inoculant alloy is designed to be the same phase as a target alloys solidification phase with a very small lattice mismatch between the two to promote solidification by epitaxial growth from the inoculants. In order to increase the survivability of these particles in the melt the alloy was designed to have a high thermal stability, achieved by refractory metal addition. Here the designed inoculant alloy was Ti-10Al-25Nb (at\%) for a $\beta$-solidifying Ti-46Al alloy. The solidifying phase for both the inoculant and melt alloys are $\beta$-Ti [12]. A small lattice mismatch was predicted (<1\%) along similar directions in each alloy, i.e. $<111>\beta_{\text {TiAl }} /<111>\beta_{\text {TiAlNb }} \sim 0.8 \%$ mismatch. This indicates that the Ti-Al-Nb alloy should work as 
an effective inoculant for the chosen alloy. As the particles added to the melt are the same phase as the solidifying alloy the particles themselves should act as centers of growth, rather than nucleation sites, and grains should grow directly from them without the need of new nuclei forming. This means the size of the particles is not critical in the same way as for traditional nucleation, rather the number of particles present for growth to occur from. Issues arising from precipitates in the solidified alloy will be avoided and the inoculants should not have any detrimental effects on the alloys final mechanical properties. The melting temperature of the inoculant was calculated with Thermo-Calc software to be $1807^{\circ} \mathrm{C}[18]$ which is much greater than the Ti-46Al melting point of $1540^{\circ} \mathrm{C}$ [5]. This indicates good thermal stability, however, the diffusional stability of the inoculants in the melt is likely lower than that of other inoculants.

\section{Experimental methods}

Both the bulk base alloy and inoculant alloy were produced from commercially pure base metals in an induction heated cold crucible apparatus. An ingot of inoculant alloy was prepared then processed into powders by cryo-milling chips obtained by drilling into the ingot. Cryo-milling was

performed in a container containing the Ti-Al-Nb chips, steel balls and air, cooled with liquid nitrogen and oscillated at up to $30 \mathrm{~Hz}$. Cryo-milling was chosen because the low temperature embrittles the particles, facilitating their breakup into smaller particles, while also preventing agglomeration. The resultant powder size distributions were determined by imaging samples of each milling time (6, 9 and 11 hours) in an SEM and measuring the particle diameters. The median particle size (D50) ranged from $163 \mu \mathrm{m}$ after $6 \mathrm{hrs}$ to $48 \mu \mathrm{m}$ after $11 \mathrm{hrs}$, the size distributions obtained can be seen in Figure 1.

[Figure 1 about here]

Once the powders had been sized pellets were produced which could then be used to introduce the particles to the melt. One gram of Ti-Al-Nb powder was mixed with one gram of aluminium powder (added as a binder to ensure the pellets retained their shape) then compressed into pellets approximately $1 \mathrm{~cm}$ diameter by $0.5 \mathrm{~cm}$ thick. Ingots of the base Ti-46Al alloy were produced by melting pellets of commercially pure Al into small ingots, grinding off the oxide, then adding them 
to commercially pure $\mathrm{Ti}$ to produce $\sim 40 \mathrm{~g}$ ingots of $\mathrm{Ti}-45.5 \mathrm{Al}$. The final $0.5 \mathrm{at} \%$ of $\mathrm{Al}$ was added to the alloy as the binder in the inoculating pellet. The bulk alloy ingots were remelted a minimum of six times to ensure homogeneity. A sample was also prepared where no powders were added but with the same final composition as the inoculated alloys (Ti-46Al-0.4Nb), i.e. the effect of the $\mathrm{Nb}$ alloying addition when not performed by inoculation, or if the inoculants were fully dissolved. To inoculate the ingots the inoculating pellet was held in the experimental apparatus away from the bulk alloy with a vacuum tube. Once the Ti-Al alloy was fully molten the vacuum was turned off and the pellet dropped into the melt. An interaction time of 20 seconds at $1580^{\circ} \mathrm{C}$ was maintained before the alloy was allowed to cool so the powders could reach thermal equilibrium and interact with the melt. The interaction time was chosen to be long enough for the particles to not have a cooling effect on solidification ( $\sim 10$ s to reach thermal equilibrium) and disseminate throughout the ingot without fully dissolving. Once cooled to room temperature the inoculated ingot was removed from the apparatus, cut, polished, and etched with Krolls reagent, then imaged with optical and electron microscopy. This procedure was repeated for each of the produced particle size distributions. Additionally the final compositions of each ingot are shown in Table 1, it is important to note the composition does not change significantly between the ingots as changes in content can drastically effect the structure of alloys on the peritectic plateau [19]. The inoculated ingots have a slightly lower aluminium content than that of the reference, so the degree of grain refinement is somewhat increased since as the aluminum content decreases on the peritectic plateau the grain size increases.

[Table 1 about here]

Equiaxed grain sizes were evaluated using backscattered electron imaging in an SEM. Micrographs were taken from the equiaxed zone of the ingots. Evaluating the grain size of equiaxed dendrites is difficult as the line intercept method cannot be used due to their 3D nature and complex shape. To avoid erroneous measurements only dendrites which appeared to be caught in cross section by the micrographs were considered, necessitating individually measuring each dendrite. The average of the lengths and widths of these equiaxed dendrites were taken as the equiaxed grain size for the sample. This method limits the total number of measurements possible but ensures measurements are representative of whole grains, not subsections, and can be used for 
both dendritic and globular structures allowing fair comparisons of grain sizes regardless of equiaxed morphology. Individual measurement reduces the total number of measurements possible but ensures each measurement represents a real, physical, dimension of a grain. The equiaxed area fraction was also evaluated by stitching optical micrographs together into a complete macrograph of the ingot cross section. The columnar/equiaxed transition could be seen and a corresponding equiaxed fraction measured.

\section{Results and discussion}

Figures 2(a) and (b) show optical macrographs of the ingots and SEM micrographs of the equiaxed zone in samples produced without inoculation (reference ingot) and with the addition of 9 hour cryo-milled Ti-Al-Nb powders. As can be seen from the figures, inoculation of the Ti-46Al alloy results in a reduced grain size and an increase of the equiaxed zone. Micrographs of the reference ingot and ingots inoculated with milled powders can be seen in Figures 3(a-d) on which false colour was applied via image processing to facilitate differentiation between grains. The morphology of the equiaxed grains changes from dendritic in the reference sample to more globular when inoculants were used.

[Figures 2\&3 about here]

The number of inoculant particles added to each ingot depended on the size distribution used since the mass of inoculant introduced remained constant in each trial. Longer milling times resulted in particles with smaller sizes and thus a larger number introduced to the melt. The number of inoculants introduced can be found by scaling the number used to determine the distribution $(\sim 10$ $\mathrm{mg})$ up to the amount introduced $(\sim 1 \mathrm{~g})$. For each milling time, the mass of powder was converted into the number of particles introduced to the melt: $4.99 \times 10^{4}, 9.91 \times 10^{4}$, and $7.63 \times 10^{5}$ particles for the 6,9 and 11 hour milled samples respectively.

Figures 4(a) and (b) show the evolution of the grain size and the equiaxed area fraction, defined as the ratio between the area of the equiaxed zone and the total cross sectional area of the ingot. The reference sample has large equiaxed grain size, $696 \mu \mathrm{m}$, while the inoculated samples have 
equiaxed grains which are much smaller, ranging from 188 to $446 \mu \mathrm{m}$. The slight reduction in grain size in the Ti-46Al- $0.4 \mathrm{Nb}$ sample $(587 \mu \mathrm{m})$ is caused by the growth restriction effect, which is a secondary effect of $\mathrm{Nb}$ additions where the partitioning of the solute element during solidification slows the growth of particles allowing more nuclei to form and thus reducing the grain size [20]. It can be seen in Figure 4, where the average grain sizes are displayed with the standard deviation of the measurements as the error, that adding inoculants reduces the grain size more than possible from just the effect of the $\mathrm{Nb}$ alloying addition and as the number of particles added to the melt increases the resultant grain size decreases. Large error bars represent a large inhomogeneity in the size of the equiaxed grains. This decreases as the grain size decreases both because the inhomogeneity in size decreases and because more grains can be measured as more grains are cut in cross section by virtue of more grains existing in the equiaxed zone. The equiaxed fraction of the ingots was also increased in all inoculated samples, as shown in Figure 4(b). The equiaxed fraction is smaller for the sample inoculated with powder milled for $11 \mathrm{~h}$, however, this sample also exhibits the smallest grain size. This could be due to the size distribution of inoculant particles. Since more small particles are present in the distribution a larger fraction of them may dissolve into the melt, leading to a smaller number of surviving particles than introduced, leading to a later occurrence of the columnar-to-equiaxed transition. It can however be stated that the inoculant powders were well mixed in the ingot due to the stirring from induction heating. The powders were present in the bulk of the molten ingot when solidification began. As cooling and solidification progressed the inoculant powders facilitated the creation of grains throughout the ingot including in front of the columnar zone. This results in a larger equiaxed fraction since equiaxed grains can be formed earlier during solidification than possible without the inoculant particles.

[Figure 4 about here]

Additionally, the inoculant particles were not observed in the solidified ingots. It would be expected that particles which acted as nucleants for equiaxed grains would be found in the center of the grains which they nucleated. As no particles were observed in the ingots it would be assumed that full dissolution occurred and the particles did not successfully nucleate any grains. The decrease in equiaxed grain size in the inoculated samples compared to the sample alloyed with $\mathrm{Nb}$ 
precludes this assumption. Particles then did survive until solidification, however their detection is difficult as they are the same $\beta-T i$ phase as the solidifying material and the relatively fast diffusion of $\mathrm{Nb}$ weakens the appearance of $\mathrm{Nb}$ rich zones. This was also the case in the previously mentioned investigation using pure $\beta$-Ti powders [17]. If the particles are acting as nuclei themselves rather than sites for heterogeneous nucleation to take place, as in traditional inoculation, it would also complicate their location as the inoculant/matrix interface would be blurred.

Further insight can also be gained by comparing the number density of particles present in the melt with the number density of grains formed during solidification. The grain density can be estimated easily from the mean grain diameters and assuming the grains formed are spherical. The number density of particles was found by assuming all the particles introduced to the melt were located in the equiaxed zone of the ingot. The columnar zones were nucleated from the crucible walls and were smaller in the ingots processed with Ti-Al-Nb particles so it stands to reason that the particles were not assisting their nucleation or growth as much and thus were likely less present there. This assumption results the greatest possible number density of particles in the equiaxed zone which in turn gives the minimum possible number of grains each particle is responsible for producing.

Figure 4 compares the number density of particles to the alloys grain density in the equiaxed zone, both expressed in $\# / \mathrm{cm}^{3}$. It can be seen in Figure 5 that the resultant grain density is much greater at a given particle density for the Ti-Al-Nb particles than found using traditional inoculants (borides) by Quested in $\mathrm{Al}$ [21] and by Gosslar in Ti-Al [22]. In Figure 5, the 1:1 ratio means that each introduced particle leads to one grain being formed in the cast alloy.

[Figure 5 about here]

It is important to note that the ratio of grain density to particle density is greater than 1:1 for the $\mathrm{Ti}-\mathrm{Al}-\mathrm{Nb}$ additions whereas in the traditional inoculation cases investigated it remains far below 1:1. This is related to the process of nucleation in traditional inoculation where at a given undercooling particles smaller than a critical diameter are not participating in the nucleation of new grains. This means at a given undercooling a portion of the distribution is not active in 
nucleation. In contrast, if the Ti-Al-Nb particles are acting as formed nuclei and centers of growth rather than nucleation, they are less dependent on being "activated" by the undercooling and each particle introduced may participate in solidification. This would explain a grain density to particle ratio of one for all distributions, however, this is not the case. The ratio decreases as the milling time increases (decreasing particle size) and is greater than one in each case. This can potentially be explained by two phenomena. First, in powders which have been milled for less time more cracks were observed in the powders than those milled for longer periods. As milling time increases the cracks which form in the powders grow and eventually split each particle into multiple smaller particles. This same breakup can occur in the cracked particles upon introduction to the melt as liquid alloy ingress into the crack causes localized increase in dissolution along the crack direction. This would result in more, smaller particles present during solidification than measured at room temperature before introduction to the melt. Additionally, since the particles are polycrystalline preferential dissolution along grain boundaries could also result in a greater number of particles being present in the melt than introduced [23]. These processes are counteracted by the full dissolution of smaller particles into the melt which then contribute to grain refinement only by the solutal growth restriction of their niobium content. The distribution of particles present in the melt is then in flux, as small particles dissolve larger ones are breaking up, making a precise evaluation of their number density on solidification difficult, however, even using the introduced number of particles to calculate their number density results in more than one grain being formed by each particle, which is significantly more than the, at best, three particles needed to form a single grain by Quested [21].

\section{Conclusions}

In conclusion, the new concept of isomorphic self-inoculation of an alloy by inoculant powders of the same phase was successfully validated using $\beta$-solidifying TiAl alloys and inoculants in the Ti-Al-Nb system. Grain size reductions by up to a factor of three were observed, while also increasing the equiaxed fraction found in the ingots. The most important factor in the effectiveness of the inoculation trials was found to be the number of particles introduced and not the size of the particles as in classical inoculation. The exact number of particles which can be active in solidification is difficult to determine. Two opposing phenomena act on the number of particles, 
while small particles may dissolve completely into the melt larger particles break apart into multiple particles. The breakup is especially important as each inoculant particle introduced formed more than one grain a piece.

\section{Acknowledgements}

JRK would like to thank Région Lorraine for co-funding his $\mathrm{PhD}$ thesis. This work was also supported by the French State through the program "Investment in the future" operated by the National Research Agency (ANR) and referenced by ANR-11-LABX-0008-01 (LabEx DAMAS).

\section{References:}

[1] D.G. McCartney. Int. Mater. Rev., 34 (1989), 247.

[2] J.A. Dantzig, M. Rappaz. Solidification. CRC press, 2009.

[3] G.I. Toth, G. Tegze, T. Pusztai, L. Granasy. Physical Review Letters, 108, 025502 (2012), 1.

[4] Y.W. Kim, D.M. Dimiduk. JOM, 43, 8 (1991), 40.

[5] V.T. Witusiewicz, A.A. Bondar, U. Hecht, S. Rex, T.Y. Velikanova. J. Alloys Compd., 465, 1-2 (2008), 64.

[6] W.G. Burgers. Physica, 1 (1934), 561.

[7] M.J. Blackburn. In The Science Technology and Applications of Titanium (London 1968), 633.

[8] S. Guide. PhD. Thesis Ecole des Mines, Nancy, 2006.

[9] A. Sankaran, E. Bouzy, J.J. Fundenberger, A. Hazotte. Intermetallics, 17 (2009), 1007.

[10] X. Wu, D. Hu. Scripta Materialia, 52 (2005), 731.

[11] M.E. Hyman, C. McCullough, J.J. Valencia, R. Levi, C.G. Mehrabian. Met. Trans. A, 20 (1989), 1847.

[12] V.T. Witusiewicz, A.A. Bondar, U. Hecht, J. Zollinger, L.V. Ya, T. Velikanova. Journal of Alloys and Compounds, 474 (2009), 86.

[13] D. Gosslar, R. Gunther, U. Hecht, C. Hartig, R. Bormann. Acta Materialia, 58 (2010), 6744.

[14] M.E. Hyman, C. McCullough, C.G. Levi, R. Mehrabian. Met. Trans. A, 22 (1991), 1647.

[15] C.J. Cowen, C.J. Boehlert. Metallurgical and Materials Transactions A, 38A (2007), 26. 
[16] T.E. Quested, A.L. Greer. Acta Materialia, 52 (2004), 3859.

[17] M.J. Bermingham, S.D. McDonald, D.H. St John, M.S. Dargusch. Phil. Mag., 90, 6 (2010).

[18] J.O Andersson, T. Helander, L. Hoglund, P. Shi. Calphad 26 (2002) 273.

[19] N.T. Reilly, B. Rouat, G. Martin, D. Daloz, J. Zollinger. Intermetallics, 86 (2017), 126.

[20] M.J. Bermingham, S.D. McDonald, K. Nogita, D.H. St. John, M.S. Dargusch. Scripta Materialia, 59 (2008), 538.

[21] T.E. Quested. PhD. Thesis University of Cambridge, 2004.

[22] D. Gosslar, R. Gunther, C. Hartig, R. Bormann, J. Zollinger, I. Steinbach. Materials

Research Society Symposium Proceedings, 1128 (2009), 91.

[23] T.E Hsieh, R.W. Balluffi. Acta Metallurgica, 37, 6 (1989), 1637. 


\section{List of Figures}

Figure 1: Ti-Al-Nb inoculant particle size distributions after (a) 6 hours, (b) 9 hours, and (c) 11 hours of cryo-milling.

Figure 2: Optical macrographs of ingot cross sections with columnar/equiaxed transition indicated as dashed line for (a) reference ingot and (b) ingot inoculated with 9 hour milled particles

Figure 3: representative SEM BSE false coloured micrographs showing equiaxed structure of (a) reference sample as well as samples inoculated with Ti-Al-Nb particles milled for (b) 6 hours, (c) 9 hours and (d) 11 hours.

Figure 4: Effect of introduced number of inoculant particles on (a) equiaxed grain size and (b) equiaxed area fraction.

Figure 5: Effect of number density of particles on grain density for Ti-Al-Nb inoculant particle additions as well as typical values for traditional inoculation found in literature. 

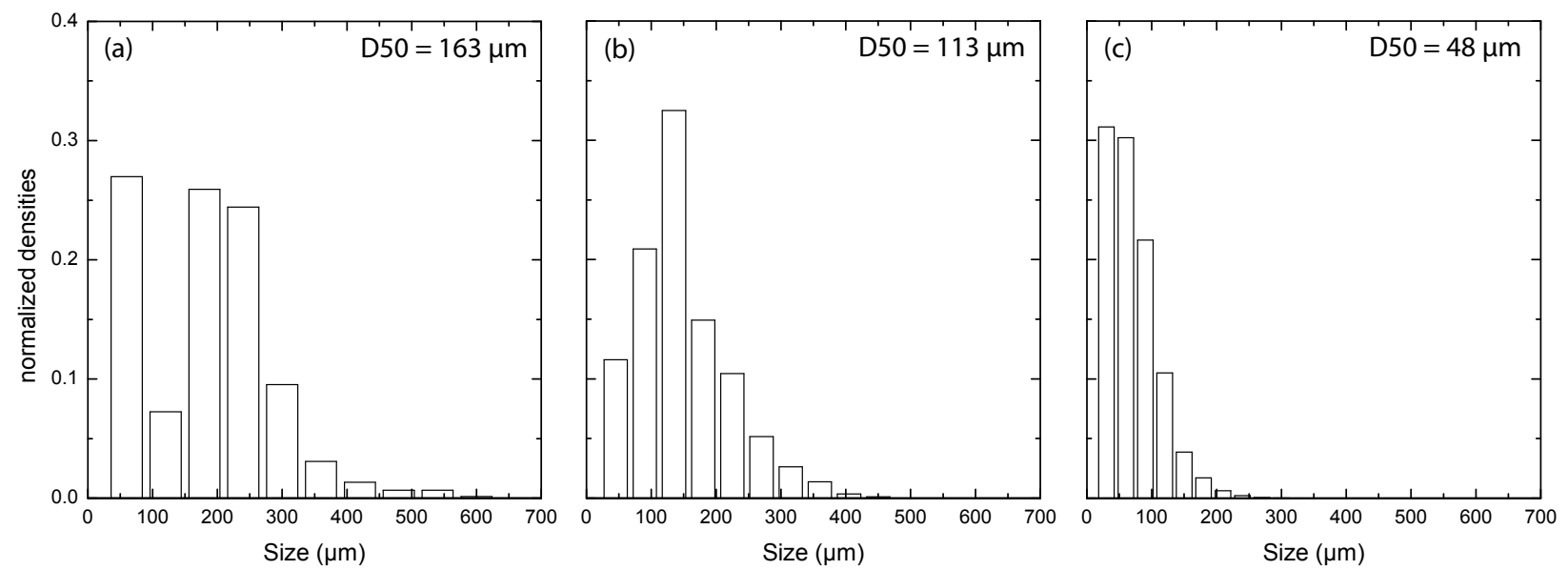

Figure 1: SEM obtained Ti-Al-Nb Ground Particle Distributions after (A) 6 hrs, (B) 9 hrs and (C) $11 \mathrm{hrs}$. The cumulative distributions are shown as dashed lines and the vertical lines are the distribution D50. 

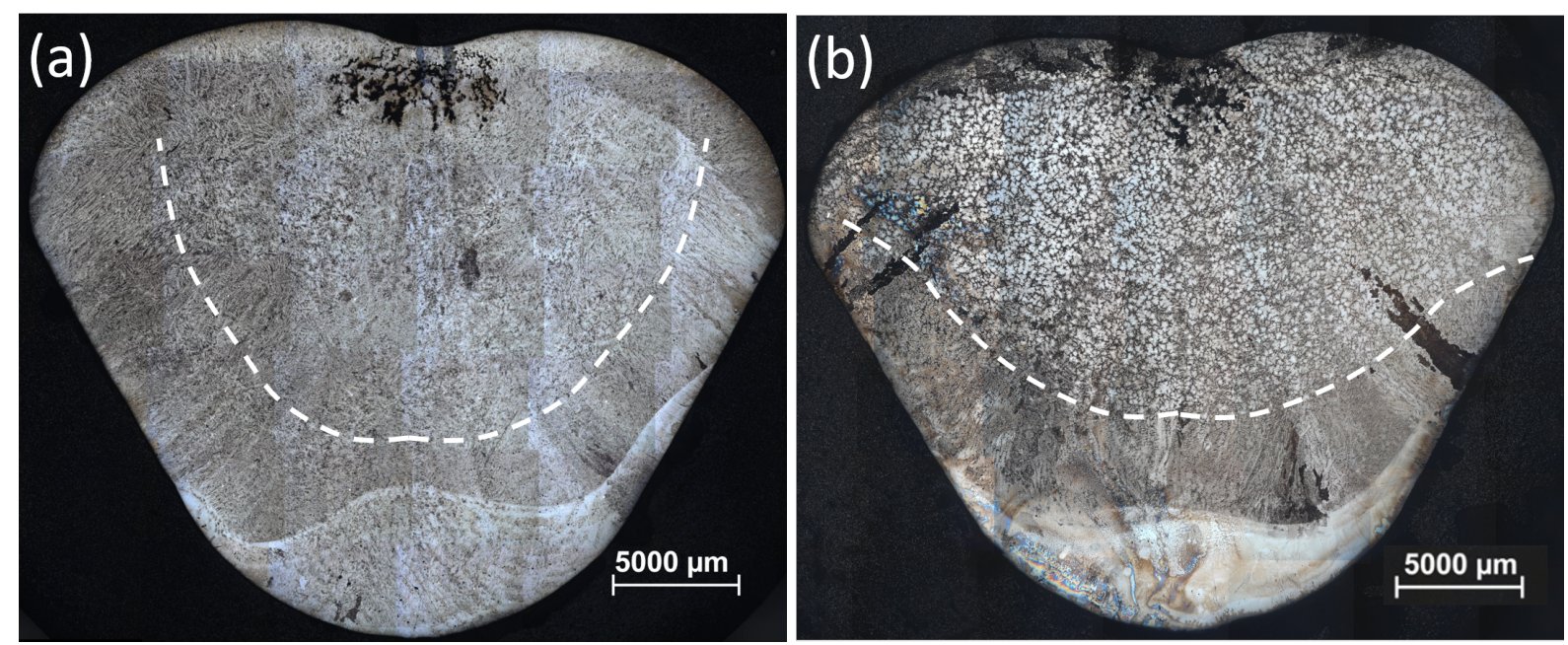

Figure 2: Optical macrographs of ingot cross sections with columnar/equiaxed transition indicated as dashed line for (a) reference ingot and (b) ingot inoculated with 9 hour milled particles 

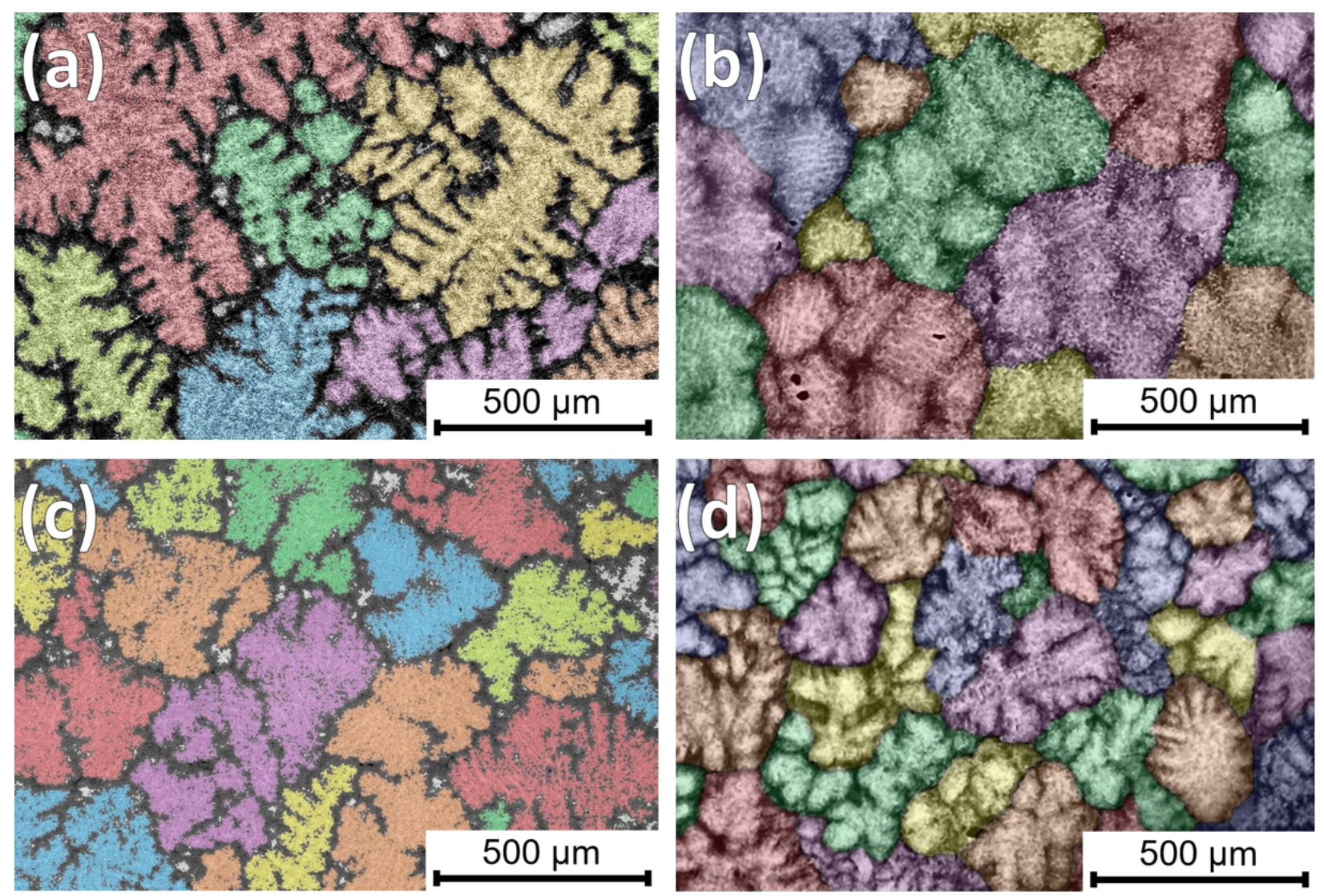

Figure 3: representative SEM BSE false coloured micrographs showing equiaxed structure of (a) reference sample as well as samples inoculated with Ti-Al-Nb particles milled for (b) 6 hours, (c) 9 hours and (d) 11 hours. 

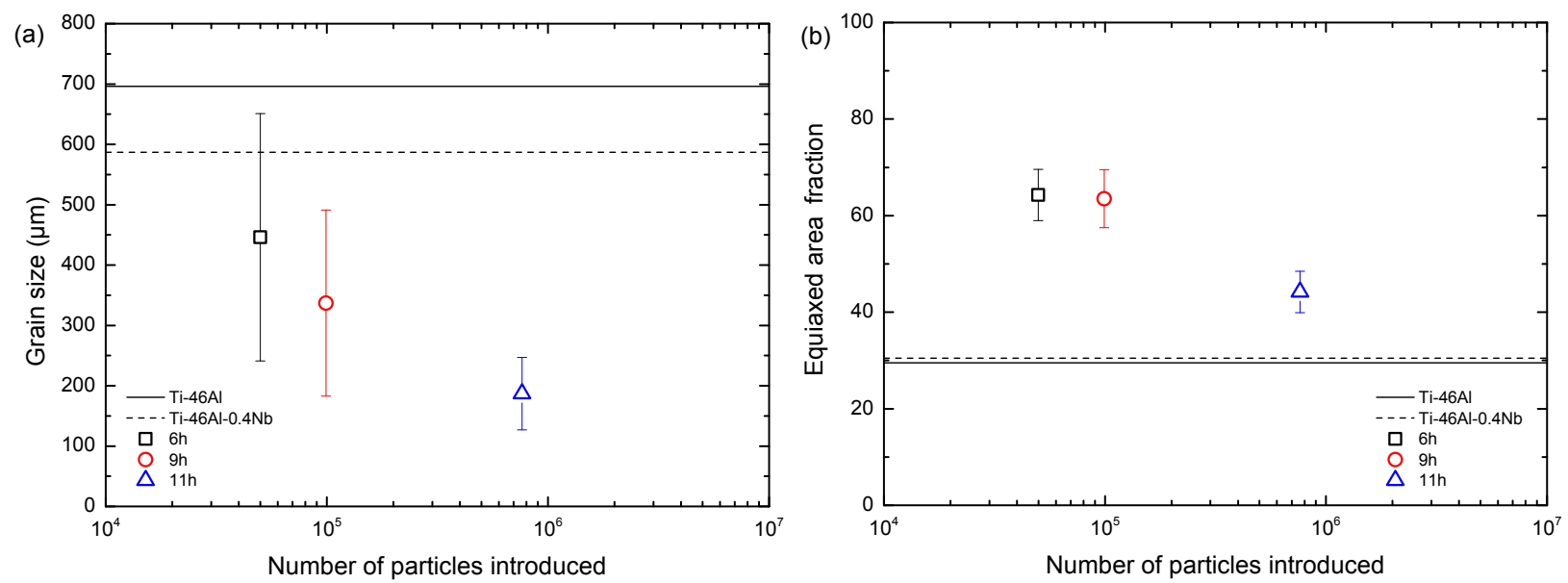

Figure 4: Effect of introduced number of inoculant particles on (a) equiaxed grain size and (b) equiaxed area fraction. 


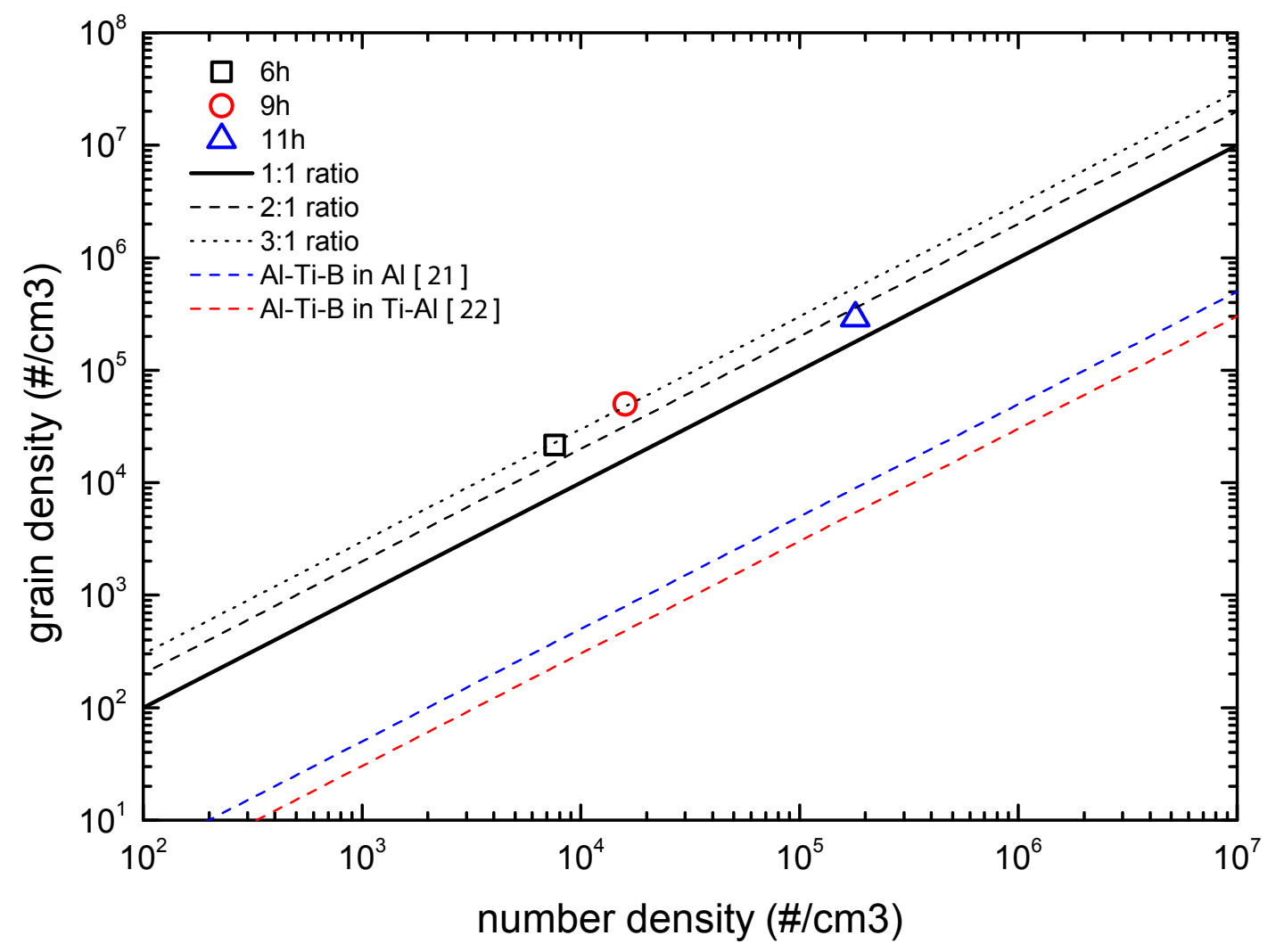

Figure 5: Effect of number density of particles on grain density for Ti-Al-Nb particle additions as well as typical values for traditional inoculation found in literature [21, 22]. 
Table 1: Ingot compositions and mass for each ingot as well as target values

\begin{tabular}{|c|c|c|c|cccc|}
\cline { 2 - 8 } \multicolumn{1}{c|}{} & Target & Reference & Target & Solutal & 6 hour & 9 hour & 11 hour \\
\hline Al (at\%) & $\mathbf{4 6}$ & 46.1 & $\mathbf{4 6}$ & 45.1 & 44.4 & 45.4 & 45.1 \\
Nb (at\%) & $\mathbf{0}$ & 0 & $\mathbf{0 . 4}$ & 0.4 & 0.4 & 0.4 & 0.5 \\
\hline Ingot Mass (g) & $\mathbf{4 0}$ & 39.9 & $\mathbf{4 0}$ & 40.6 & 40.9 & 39.2 & 38.4 \\
\hline
\end{tabular}

